\title{
Cáncer de mama y osteoporosis
}

\author{
Jorge P. Suárez*; William Onatra H.**; Jacinto A. Sánchez**
}

\begin{abstract}
RESUMEN: Se presenta un caso de una mujer de 54 años, con antecedentes de mastectomía izquierda por Ca. canalicular Estado III, con Osteoporosis 0 que no ha respondido a la terapia a base de calcio y fluor, proponiéndose la posibilidad de iniciar terapia hormonal de reemplazo.
\end{abstract}

SUMMARY: A 54 years old woman's case is presented with left mastectomy antecedents by Ca. canalicular state III, with an 0 osteoporosis which has not responded to the therapy based on calcium and fluorine, suggesting the possibility of beginning a replacing hormonal therapy. Absent in $\mathbf{9 6 . 7 \%}$ of patients: just in one case it was neccesary to suspend the medicament because of a severe fibro-cystic disease in the breast. The rest of complications cannot only be directly atributed to the use of estrogens. It is concluded that substitution therapy combined with progestational-estrogen during climacteric is accurate.

\section{Presentación de Caso Clinico}

$\begin{array}{ll}\text { Nombre } & : \text { B.M. } \\ \text { Edad } & : 47 \text { años } \\ \text { Natural } & : \text { Coyaima. Tolima } \\ \text { Procedente } & : \text { Bogotá } \\ \text { E.Civil } & : \text { Soltera }\end{array}$

Fecha de Ingreso: Abril 28 de 1987

$\begin{array}{ll}\text { HC } & : 523277 \text { IM } \\ \text { Raza } & : \text { Mestiza } \\ \text { Ocupación } & \text { : Hogar } \\ \text { Religión } & \text { : Católica }\end{array}$

\section{Motivo de Consulta}

Oleadas de calor de 1 año de evolución, acompañada de decaimiento, cefalea y sudoración.

\section{Enfermedad actual}

Se inicia en agosto de 1985 cuando fue mastectomizada en el Instituto Nacional de Cancerología por un Ca. de mama izquierda. Recibió quimioterapia a base de CY, MTX y $5 \mathrm{~F}$ por 6 ciclos, Cobaltoterapia quedando como secuela un linfedema obstructivo post irradiación en Miembro Superior Izquierdo.

\footnotetext{
* $\quad$ Residente III, Programa de postgrado, Departamento de Obstetricia y Ginecología, Universidad Nacional de Colombia.

** Profesor Asistente, Departamento de Obstetricia y Ginecología, Universidad Nacional de Colombia, Clínica de Menopausia, Instituto Materno Infantil - Santafé de Bogotá.
}

\author{
Antecedentes \\ Familiares. \\ Madre Hipertensa. Cáncer, Diabéticos Negativos. \\ Personales. \\ - Infancia. Vacunas positivas, contacto con perros y \\ gatos. Hepatitis no. \\ - Médicos: Bronquitis a repetición.Ca. mama en \\ control. \\ Traumáticos: Linfedema post radiación MSIz- \\ quierdo \\ Quirúrgicos: Amigdalectomía en 1982. Ooferec- \\ tomía Derecha 1976. Mastectomía Izquierda 1985. \\ Bartholinectomía 1986. \\ Alérgicos: Penicilina. \\ Tóxicos: Negativo \\ Fármacos: Ibuprofen
}

\section{Gineobstétricos:}

Menarquia: 16 años. Telarquia 11 años, Pubarquia 14 años. Ciclos 30 x 3 UR:12 Octubre de 1985. Go Po Flujo Blanco pruriginoso. Citología vaginal Octubre 86 A2.

Exámen Físico: Paciente en buen estado general, con edema en M.S. Izquierdo.TA; 120x 80 m.m. Hg. FC 72 x min. Fr 16x min.

Peso $75 \mathrm{Kg}$. Talla $1.52 \mathrm{mts} . I \mathrm{MC}$; Env. $1.70 \mathrm{mts}$.

Orofaringe: Protesis Superior;

Cardiopulmonar:cicatriz de mastectomía izquierda.R. Cardíacos dentro de límites normales. Ventilación normal en ambos campos.

Abdomen: blanco sin visceromegalias. Cicatriz mediana infraumbilical.

Extremidades: Linfedema MSIzquierdo. No várices.

Ginecológico: Seno derecho; normal. Genitales externos normales. Sin celes, ni incontinencia urinaria. 
Figura $1 \mathrm{~A}$

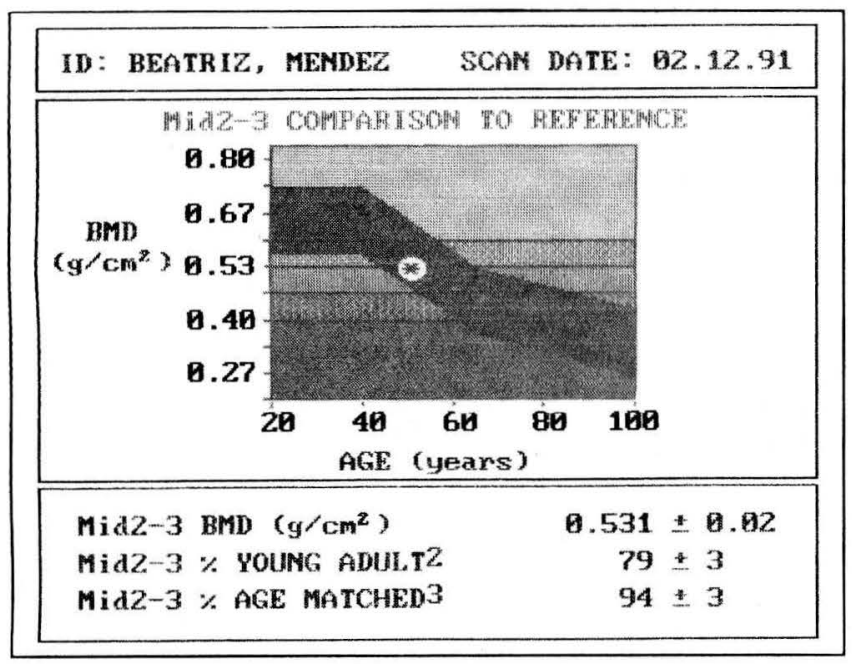

Figura $2 \mathrm{~A}$

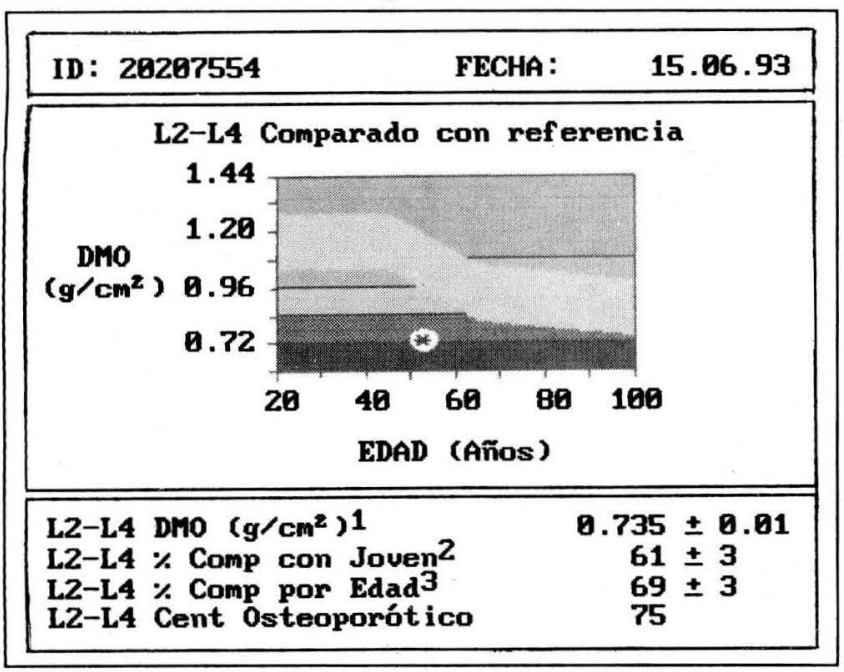

Cérvix de nulipara, flujo blanco. TV: útero en AVF dentro de límites normales. Anexos negativos.

ID: Síndrome climatérico. Antecedentes de Mastectomía Izquierda por Ca de mama en control por INC.

Evolución: Se inicia terapia con Veralipride $20 \mathrm{mg}$ al día por 3 meses. Durante este tiempo se confirma una Diabetes Tipo II fase II controlada en el Hospital San Juan de Dios de Bogotá.

Radiografía de Columna muestra Osteopenia generalizada agosto 3 del 87 . Se inicia terapia a base de calcio $600 \mathrm{mg} /$ día más Fluoruro $20 \mathrm{mg}$ en forma cíclica. Se recomienda dieta rica en calcio y programa de ejercicio que no se lleva a cabo por problema de horario en el gimnasio.

En 1991 se realiza una Densitometría que muestra «valores satisfactorios de densidad ósea en el esqueleto axial y apendicular». En esas condiciones continúa su terapia con Calcio continuo por 3 meses, Fluor 3 meses y 3 meses de descanso.
Figura $1 \mathrm{~B}$

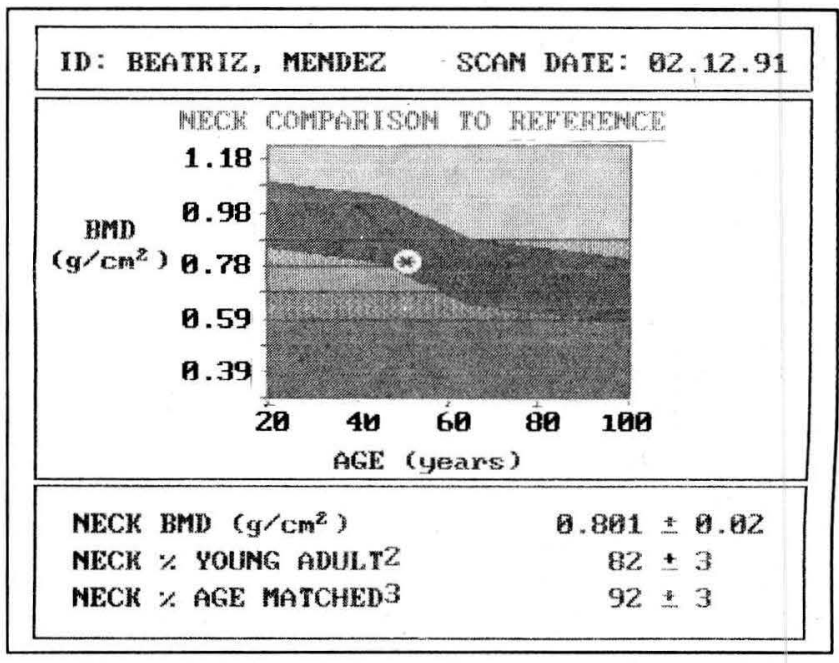

Figura 2 B

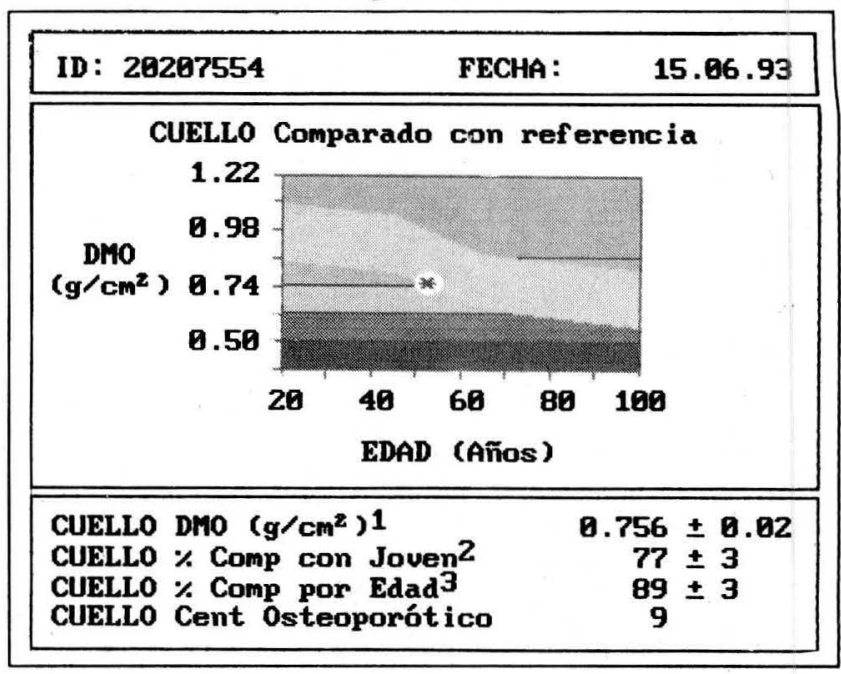

Control realizado en junio del 93, muestra una disminución del contenido de calcio a nivel de columna (69\%) con aumento del riesgo de fractura y en cuello femoral ( $89 \%$ ) dentro de límites normales. Se plantea la necesidad de cambiar la terapéutica y se solicita concepto por INC donde informa que se trata de una $\mathrm{Ca}$ canalicular infiltrante grado III, sin dato de receptor para estrógenos y contraindican la terapia de sustitución hormonal (THS).

Concepto Clínica Climaterio IMI: Se podría cambiar a la luz del conocimiento actual THS.?

Exámenes Paraclínicos:

febrero 2 86: Grupo 0 (+) Serología: no reactiva Cuadro Hemático:

Febrero 2 86; Htto 40 HB 13.7 Leuco 7.200

Octubre 29 87: Htto $40 \mathrm{Hb} 12.8$ Leucocitos 4.750

Enero 21 90: Htto $40 \mathrm{Hb} 13.7$ Leuco 5.800

Enero 25 94; Htto $42 \mathrm{Hb} 13.4$ gr Leucocitos 6.100

Química sanguínea; 


\section{Glicemia}

Febrero 2 86: $88.4 \mathrm{mg} \%$

Febre 6 90: $130 \mathrm{mg} \%$

Marzo 3 93: 110

Septiembre: 7 93: $134 \mathrm{mg} \%$

Marzo 15 94: $129 \mathrm{mg} / \mathrm{dl}$.

Enero 25 94: $115 \mathrm{mg} \%$

Proteínas Totales:

Febrero 2 86: 8.0 Alb 5.4 Glob 2.6

Novi $2587 ; 8.0$ alb 5.4 glob 2.6

Jun 290 Prote 8.9 alb 6.0 glo 2.0

\section{Colesterol}

Febrero 2 86: f306 mg\%

Marzo 20 90; $263.4 \mathrm{mg} \%$

Enero 21 91: $219 \mathrm{mg} \%$

Febrero 2 93: $236 \mathrm{mg} \%$

Fosfatasa alcalina

Octubre 16 91: 39 U/l (115.250)
Junio 19 93: 246 U/L (50-250)

Marzo 14 94: 174 U/L (60-300)

Rx Columna dorso lumbar

Mayo 10 86; Mínima escoliosis lumbar. Osteopenia generalizada.

Marzo 23 90: Mínima osteopenia.

Densitometría

Dic 2 91: Columna lumbar $0.531 \mathrm{gr} / \mathrm{cm} 274 \%$ para la edad. Cuello femoral $0.801 \mathrm{gr} / \mathrm{cm} 283 \%$ para la edad

Jun 15 93:Columna Lumbar $0.735 \mathrm{gr} / \mathrm{cm} 269 \%$ para la edad. Cuello Femoral $0.756 \mathrm{gr} / \mathrm{cm} 289 \%$ para la edad

Citología Vaginal

$\begin{array}{ll}\text { Octubre } & 2986 \mathrm{~A} 2 \\ \text { Febre } & 2888 \mathrm{~A} 2 \\ \text { Julio } & 2391 \mathrm{~A} 2 \\ \text { Enero } & 2193 \mathrm{~A} 2 \\ \text { Enero } & 2594 \mathrm{~A} 2\end{array}$

\section{CONCEPTOS CLINICOS}

\section{1) BERNARDO MORENO ESCALLON*}

A la luz de los conceptos ortodoxos sobre la contraindicación absoluta para el uso de Estrógenos en pacientes con cáncer de glándula mamaria, habría que partir de ese principio fundamental, y luego analizar los riesgos y beneficios de una terapia hormonal y también analizar el costo-beneficio de otras terapias alternativas.

Si el paciente tuvo una neoplasia de Seno estado III tratada, sin estudio de receptores estrogénicos, con historia familiar de cáncer y la paciente aún conserva la glándula mamaria contralateral, aunque no haya estudio de receptores para estrógenos, hay que asumir que los podría tener positivos en un $50 \%$ de las probabilidades. Estos argumentos hacen que exista una contraindicación para el uso de estrógenos. Como la paciente se encuentra en manopausia y tiene un proceso osteoporótico en la columna con aumento de riesgo de fractura, es necesario optar por otras terapias alternativas, que podrían ser:

1- Bisfosfonatos: Pertenecen a una nueva familia de fármacos desarrollados en las dos últimas décadas, y se han encontrado útiles en el tratamiento de una variedad de desórdenes óseos, de los dientes y metabolismo del calcio. Entre estos compuestos el Etidronato, es el único compuesto en forma oral disponible en los USA. Su principio de acción es logrando una reducción global del recambio óseo, disminuyendo la actividad osteoclástica, lo cual conduce a un aumento de la masa ósea. Trabajos

* Clínica Fray Bartolomé de las Casas, Caja de Previsión Social Distrital (CPSD), Unidad de Ginecología Santafé de Bogotá. realizados en Copenhagen en 66 mujeres con osteoporosis post-menopáusica durante 3 años y otro realizado en forma multicéntrica en 429 mujeres post-menopáusicas en USA durante dos años, demostraron que el uso del Etidronato en esas pacientes aumentó significativamente el contenido óseo de la columna vertebral y redujo la incidencia de fracturas vertebrales. El Etidronato se recomienda en dosis de $400 \mathrm{mgs} /$ día durante 14 días cada 3 meses. Para complementar el incremento en la masa ósea, es esencial tomar Calcio oral el cual sumado al de la dieta debe ser alrededor de 1.500-2.000 mgrs/día (1).

2- Calcitonina: Es una hormona peptídica que inhibe la resorción ósea mediante una acción directa sobre los osteoclastos. Detiene la pérdida de masa ósea en pacientes con osteoporosis sintomática. Se utiliza por vía parenteral o por vía intranasal o dosis de 50-100 UI/día (2-3). La vía parenteral no es práctica en tratamientos largos, ya que hay que utilizarla 2 a 3 veces por semana. La vía intranasal esta siendo utilizada recientemente y no se conocen efectos a largo plazo. Además su costo es una limitante para uso generalizado.

3. El fluoruro sódico el cual estimula la proliferación osteobiástica y aumenta la velocidad de formación osea. También ha sido utilizado en el tratamiento de la osteoporosis establecida. Tiene buena acción sobre el hueso trabecular como el de la columna vertebral. La respuesta a veces no es notoria antes de 12 a 24 mesếs y en un $30 \%$ de los pacientes no hay una respuesta positiva $(3,4)$. En este caso clínico específico la paciente había recibido fluoruro durante varios años y en los últimos meses se estaba observando una respuesta refractaria. 
4. Tamoxífeno y Acetato de Medroxiprogesterona: Los progestágenos utilizados en combinación con los estrógenos, además de prevenir el cáncer de endometrio, reducir el riesgo de carcinoma de seno, promueven la formación osea (5) y reducen la resorción osea en una forma equivalente a $0.625 \mathrm{mgrs}$ de estrógenos conjugados (6). Teniendo en cuenta el efecto antiestrogénico del Tamoxifeno sobre el seno y estrogénico leve en otros tejidos, combinado con el efecto positivo sobre el hueso del acetato de madroxiprogesternona, podría ser una combinación menos costosa, que otros tratamientos y otra alternativa por analizar a largo plazo con esta paciente.

Finalmente, a esta paciente además del tratamiento farmacológico, se recomendaría en lo posible reducir su peso al máximo, para aliviar toda esa tensión física sobre la columna que se encuentra en riesgo de fractura.

Valdría la pena monitorizar esta paciente con "Densitometría Bioquímica" mediante determinación de Priridinolina y Deoxipiridinolina. Con estos marcadores biológicos, a veces se detecta el problema antes que se manifieste en una Osteodensitometría para estudio de Osteoporosis.

\section{BIBLIOGRAFIA}

1. Christiansen C., Riis B. "Post-menopausal Osteoporosis" Fagerstien 8, 2950 Vedback Dinamarca, 1990.

2. Overgaard R. "'Effects of intranasal synthetic salmon calcitonin on bone breakdown". Br. Med. J. 1989; 299:477.

3. Current Perspectives in the Management of the Menopausal and Postmenopausal patient. Symposium. Am. J. Obstet Gynecol 1987; 156: 1353 .

4. Hodsman B., Drost D. "The response of vertebral bone mineral density during the treatment of

5. Gambrell R.D. Use of progestogen ther apy. Am. J. Obstet Gynecol. 1987; 156: 130-131.

6. Lobo R., Singer M. "Depo-Medroxyprogester one Acetate compared with conjugated estrogens for the treatment of Post-menopausal women. Obstet Gynecol 1984; 63:1.

\section{2) DAVID VASQUEZ AWAD*}

Se trata de una paciente relativamente joven (47 años) con antecedente de Ca de mama (canalicular infiltrante grado III) operado, irradiado y sometido a tratamiento con quimioterapia, aparentemente sin recidiva. Se presenta una osteoporosis con pobre respuesta al tratamiento con Calcio y Flúor.

Desafortunadamente no existen datos acerca de receptores estrogénicos ni tampoco se informa si la paciente ha recibido o recibe Tamoxifen. Este último dato es de capital importancia, ya que hay evidencia de que el Tamoxifen estabiliza la pérdida de hueso en un grado comparable al asociado a la administración de estrógenos

* $\quad$ Médico Ginecólogo y Obstetra, Universidad Javeriana Perinatólogo CLAP-OPS/OMS.
$(1,2)$. Igualmente, existen reportes que demuestran la capacidad del Tamoxifen para disminuir los niveles de colesterol, primariamente por disminución del colesterol LDL, pudiendo reducir el riesgo cardiovascular $(3,4)$.

Referente a la terapia estrogénica en particular, yo recomendaría prudencia en esta paciente, ya que aunque Wile y DiSaia (5) abogan por una reconsideración respecto a la prohibición de la Terapia de reemplazo estrogénico en pacientes con antecedentes de $\mathrm{Ca}$ de mama, la mayoría de los expertos son reacios a administrarla más aún como en este caso si no se tienen datos acerca de los receptores para estrógeno.

\section{BIBLIOGRAFIA}

1. Fornander R, Rutqvist LE, Sjoberg HE, et al. Long-term adjuvant tamoxifen in early breast cancer: effect on bone mineral density in postmenopausal women. J Clin Oncol 1990; 8:1019-1024.

2. Love RR, Mazess RB, Tormey DC, et al. Bone mineral density in women with breast cancer treated with adjuvant tamoxifen for at least two years. Breast Cancer Res Treat 1988; 12:279-302.

3. Barrett-Conner E, Bush Tl. Estrogen replacement and coronary heart disease. Cardiovasc Clin 1989; 19:159-172.

4. Love RR, Newcomb PA, Weibe DA, et al. Effects of tamoxifen therapy on lipid and lipoprotein levels in postmenopausal patients with node-negative breast cancer. N Natl Cancer Inst 1990; 82:1327-1332.

5. Wile AG, DiSaia PJ. Hormones and breast cancer. Am J Surg 1989; 157: 438-442.

\section{3) CLINICA DEL CLIMATERIO DEL INSTITUTO MATERNO INFANTIL}

La espectativa de vida en la mujer ha venido en aumento en las últimas décadas y para Colombia de acuerdo a sus proyecciones se espera que sea de 76 años para el aõ 2.000 , con cerca de 6 millones de pacientes mayores de 50 años.

El manejo del síndrome climatérico implica un equipo multidisciplinario y no hay duda de la mejor calidad de vida de este grupo poblacional, al ingresar alos programas de estas consultas integradas. La terapia Hormonal de Sustitución (THS) ofrece un pilar en la prevención y manejo de las diversas patologías asociadas, como las cardiovasculares, metabólicas, oseas que ocupan una de las primeras causas de morbimortalidad. Los antecedentes de patología maligna de mamam ha sido una contraindicación absoluta para la THS. (1).

Recientemente dos Meta-análisis valorando la THS con estrógenos conjugados a dosis de $0.625 \mathrm{mg}$, no encuentran asociación (1) o un riesgo aumentado luego de 15 años de uso (2). Desde hace 6 años han aparecido reportes $(2,3,4,5,6)$ de la evolución de pacientes con diagnóstico de $\mathrm{Ca}$. mamario en diversos estadios, tratados con THS y receptores (+) o (-) para estrógenos en tejido mamario y quienes habían aceptado voluntariamente este tipo de terapia. 
El estudio que más pacientes tiene es del Di Saia (8) que incluye 77 casos, edad promedio 50 años, Diagnóstico de Ca. de mama a quienes se les había hecho un seguimiento a 15 años. El intervalo entre el diagnóstico e inicio de la terapia fue de 23 meses y un seguimiento promedio de 27 meses. La mayoría recibió estrógenos conjugados y solo 13 se asocio a progestagenos. La supervivencia promedio fue de 53 meses. De las que iniciaron la THS, 71(92\%) no tenían evidencia de enfermedad, 3(4\%) habían mejorado de la enfermedad y 3 habían muerto (1 por complicaciones de la quimioterapia y las otras 2 por avance de la enfermedad). Concluye que aunque este estudio no es suficiente seguro de la utilidad de la THS en mujeres con antecedentes de Ca. mamario si demuestra que su exposición no produce recurrencia, haciendo necesario un estudio de casos y controles.

Para el caso presentado dada la escasa respuesta a la terapia y con el deseo de evitar la pérdida osea, podría iniciarse la THS, explicándole a la paciente los riesgos- beneficio de la medicación y autorización escrita de su aceptación.

\section{BIBLIOGRAFIA}

1. Dupont WD, Page Dl. Menopausal estrogen replacement therapy and breast cancer. Arch Intern Med 1991; 151:67-72.

2. Steimber KK, Thacker SB, Smith SS et all. A meta-anlysis of the effect of estrogen replacement therapy on the risk of breast cancer, JAMA 1991; 266:1362-1365.

3. Brener H, Engelsman B, Stegmaier C et all. Clinical Epidemiology of bilateral breast cancer. Cancer 1993; 72:3629-3635.

4, Wile AG, Opfell RW, Margilet DA. Hormone Replacement therapy in previously treatet breast cancer patients. Am J Surg 1993; 165:372-375.

5. Buller RE. Hormone replacement therapy following ginecologic cancer. Postgraduate Obstet Gynecol 1993; 13:1-5.

6. Creasman WT. Hormone replacement therapy in breast cancer. Lancet 1993; 342:1232.

7. Marchant DJ. Estrogen-replacement therapy after breast cancer Cancer 1993; 71:2169-2176.

8. Di Saia PJ. Hormone-replacement therapy in patients with breast cancer: a reappraisal. Cancer 1993; 71:1490-1500

\section{Correos \\ de Colombia}

\section{Adpostal}

\section{Estos son nuestros servicios ¡utilícelos!}

- SERVICIO DE CORREO ORDINARIO

- SERVICIO DE CERTIFICADO ESPECIAL

- ENCOMIENDAS CONTRA REEMBOLSO

- SERVICIO DE FILATELIA

- SERVICIO ELECTRONICO

- SERVICIO INTERNACIONAL APR/SAL

- SERVICIO RESPUESTA COMERCIAL

- SERVICIOS ESPECIALES
- SERVICIO DE CORREO CERTIFICADO

- SERVICIO ENCOMIENDAS ASEGURADAS

- SERVICIO CARTAS ASEGURADAS

- SERVICIO DE GIROS

- BUROFAX

- SERVICIO "CORRA"

- SERVICIO TARIFA POSTAL REDUCIDA

Teléfonos para quejas y reclamos: 3340304 - 3415536 - Santafé de Bogotá, D.C. - Colombia Cuente con nosotros hay que creer en los Correos de Colombia 